\title{
Nature of Chromosome Pairing in Allopolyploids of Arachis and Their Stability
}

\author{
V. S. Raman \\ Agricultural College and Research Institute, Coimbatore-3, India
}

Received August 7, 1979

Cytogenetic investigations on wild species of Arachis related to the cultivated groundnut ( $A$. hypogaea) carried thus far have brought out the existence of more than one diploid $(2 \mathrm{n}=4 \mathrm{x}=20)$ species ( $A$. diogoi, $A$. duranensis, $A$. villosa and $A$. villosulicarpa) whose genomes seem to be homologous to one of the two sets of chromosomes present in the tetraploids ( $A$. glabrata, A. hagenbeckii, A. hypogaea, $A$. marginata and $A$. monticola). The preferential autosyndesis exhibited by the experimental allotetraploid compared to the meiotic associations in the induced autotetraploids of diploid species and the differential affinity disclosed by the allohexaploids provided evidence for cryptic structural differentiation between genomes which, however, did not interfere with chromosome pairing in the diploid and triploid hybrids. The chromosome complements of the tetraploid species themselves appeared to possess no absolute dissimilarity, though differentiation was apparent. The allopolyploid nature of the 40-chromosome ( $2 n$ ) species of Arachis was indeed clear and evidence gathered tended to amplify it further as segmental alloploidy tending towards genomic allopolyploidy.

Results of inquiry directed towards increasing the variability and incorporation of desirable characteristics not available in the "gene pool" of $A$. hypogaea revealed the importance and significance of chromosome differentiation in the synthesis of new forms of groundnut. Chromosome associations in hybrids conforming to different levels of ploidy and genomic composition are reported below, the scrutiny of which focusses attention on the point that pairing can be enhanced by genetic mechanisms even to give maximum expression to residual affinity.

\section{Material and methods}

The diploid species, $A$. diogoi, $A$. duranensis and $A$. villosa and five natural tetraploids viz., A. glabrata, A. hagenbeckii, A. hypogaea, A. marginata and A. monticola served as the initial parental stock. Nine cultivars of $A$. hypogaea classified as the Spanish (TMV. 2, TMV. 7, TMV. 9, POL. 2 and OSN. 1), Valencia (Gangapuri), Virginia-bunch (TMV. 10) and Virginia-spreading (TMV. 3 and M. 13) were involved in inter-specific crosses. Autotetraploid genotypes of $A$. villosa and $A$. villosulicarpa were also employed in the synthesis of allopolyploids. In addition, diploid $(A$. duranensis $\times A$. villosa), and triploid hybrids $(A$. hypogaea $\times A$. duranensis and $A$. hypogaea $\times A$. villosa) and the allohexaploid ( $A$. hypogaea $\times A$. villosa) could be used as parents in different sets of cross pollinations. Hand emasculation 
of the flowers borne on field grown plants preceded artificial matings. Flower buds of the appropriate size were fixed in Carnoy's $(6: 3: 1)$ solution and anthers squashed in acetocarmine. Chromosome pairing was analysed in pollen mother cells (PMCs) at diakinesis and metaphase I.

\section{Results}

The pattern of chromosome pairing together with the proportion of genomes of the natural and experimental polyploids are presented in Table 1. The material comprised autotetraploids of two diploid species, the cultivated and semi-wild annuals of $A$. hypogaea and $A$. monticola respectively and the perennial $A$. glabrata and hybrids realised from parental combinations of $4 \mathrm{n} \times 2 \mathrm{n}$ and reciprocal, $4 \mathrm{n} \times$ $4 n$ and $4 n \times 6 n$. In Table 2, information on meiotic pairing relates to derivatives of crosses where one of the parents is hybrid in origin. Five parental combinations viz., $2 n \times 2 n, 3 n \times 2 n$ and reciprocal, $2 n \times 4 n$ and $4 n \times 3 n$ are cited in this Table. The progeny behaviour of experimental alloploids with three levels of ploidy is summarised in Table 3, providing data on variations in chromosome number and cytological behaviour. Chromosome pairing in PMCs of artificial alloploids (3n, $4 n, 6 n$ and $8 n$ ) is illustrated in Figs. 1-13.

\section{Chromosome pairing in natural and synthetic polyploids}

The haploid complement of the allotetraploid $(2 n=40)$ species of $A$. hypogaea has so far been recognised to comprise the $\mathrm{A}^{\mathrm{h}}$ and $\mathrm{B}^{\mathrm{h}}$ genomes each with 10 chromosomes. The genome of the diploid $(2 n=20)$ A. villosa could be indicated as

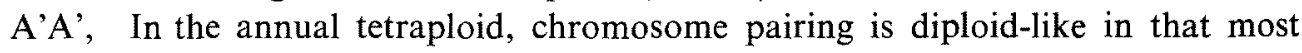
of the chromosomes pair as bivalents with the exception of a quadrivalent being noticed in a very low frequency leading to the average association of 0.05IV + 19.90II. A. monticola which closely resembles the cultivated species but is not grown in cultivation displayed 0.50IV $+19.00 \mathrm{II}$. The tetraploid species with the rhizomatous habit entailing poor seed fertility gave a maximum of 4 quadrivalents and the mean association was represented by 1.46IV $+17.08 \mathrm{II}$. Chromosome pairing in the hybrids (AA'B) of A. hypogaea (TMV. 2) with the diploid species resulted in trivalent and bivalent configurations in addition to univalents. In triploid $\mathrm{F}_{1} \mathrm{~s}$ of the cultivated groundnut with the diploids of $A$. diogoi, $A$ duranensis, $A$. villosa and $A$. villosulicarpa, trivalents ranged from 1.05 to 1.20 per cell. The allotriploid of $A$. monticola $\times A$. villosa presented 1.46 trivalents. The triploids of A. diogoi $\times A$. glabrata and A. villosa $\times A$. marginata gave 2.60 to 3.0 trivalents per cell, in contrast to 8.88 trivalents in the autotriploid of $A$. villosa. The haploid complement of the diploids appears to be more akin to one of the two genomes present in the perennial tetraploid species. In the cultivated species, association of quadrivalents is drastically reduced due to differential affinity while the truly wild tetraploid exhibited heterogenetic quadrivalents. The meiotic behaviour of the triploid hybrids is neither similar to that of a strict autotriploid nor it conforms to the pattern of chromosome pairing typical of an allotriploid, yet the segmental alloploid nature of the tetraploid species is definite. 


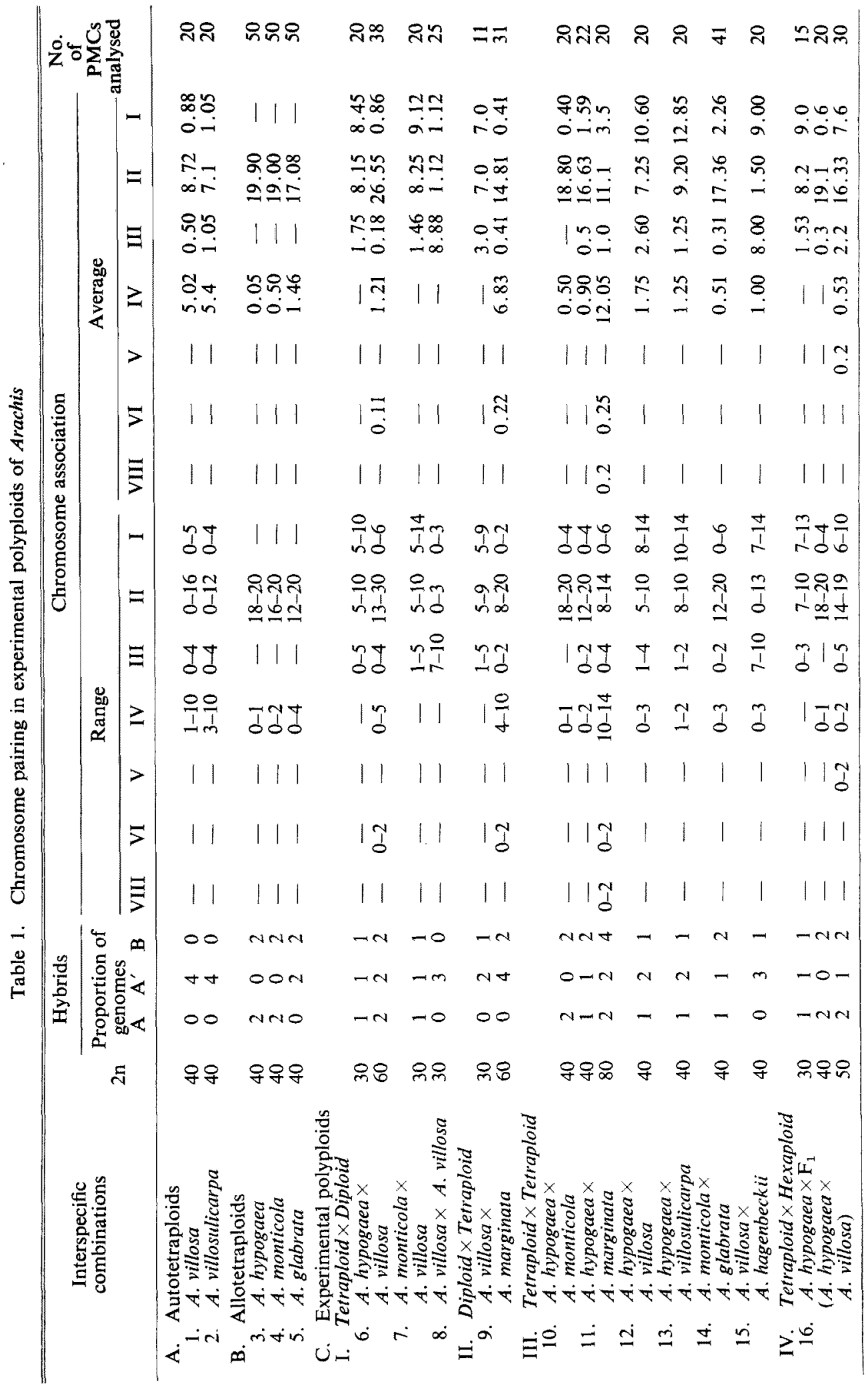




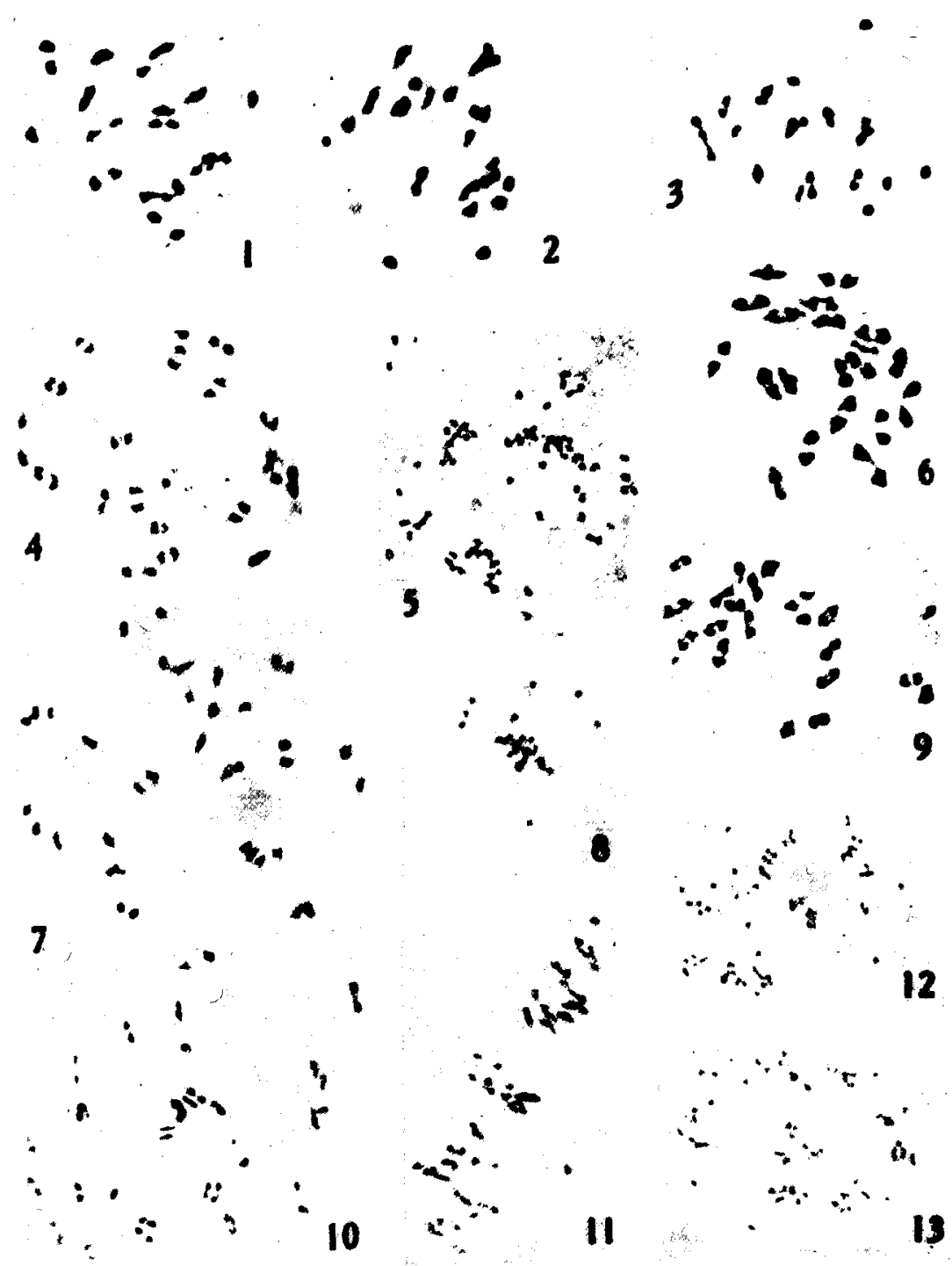

Figs. 1-13. 1, allotriploid between the Spanish groundnut (POL. 2) and A. villosa showing 10 II $+10 \mathrm{I}$ at prometaphase. 2 , triploid plant recovered in the open-pollination progeny of the allohexaploid exhibiting 1 III +7 II +13 I. 3, triploid isolated in the open-pollination progeny of the tetraploid hybrid between the Spanish cultivar (TMV. 9) and autotetraploid A. villosa; 1 III+ 9 II +9 I at prometaphase. 4, tetraploid derivative of the triploid hybrid; diakinesis showing $19 \mathrm{II}+2 \mathrm{I} .5$, aberrant behaviour of chromosomes at the second division in a tetraploid plant of the parentage, A. hypogaea $\times$ allohexaploid. 6, A. hypogaea with $20 \mathrm{II}$ at metaphase I. 7, allotetraploid plant resulting from the cross, Spanish groundnut (TMV. 9) $\times$ autotetraploid A. villosa; diakinesis with 1 IV +2 III +7 II +16 I. 8, triploid hybrid ( $A$. hypogaea $\times$ allohexaploid) showing prominently a trivalent and five univalents. 9 , tetraploid $\mathrm{F}_{1}(A$. hypogaea $\times A$. monticola); $19 \mathrm{II}+2 \mathrm{I}$ at prometaphase. 10 , and 11, allohexaploid plant ( $A$. hypogaea $\times$ A. villosa); prometaphase with $2 \mathrm{IV}+26 \mathrm{II}$ and precocious disjunction of bivalents at metaphase I. 12 and 13, natural octaploid showing bivalents and univalents in addition to multivalent associations. Figs. 1, 2 and $6 . \times 1800$. Figs. 3, 4, 7 and $9 . \times 1600$. Rest. $\times 1400$. 
The original amphitriploids (AAA' $\mathrm{A}^{\prime} \mathrm{BB}$ ) secured from the open-pollination progeny of the allotriploid (A. hypogaea $\times$ A. villosa) exhibited 30 bivalents in 26 per cent of PMCs and in the rest, multivalents prevailed in conjunction with bivalents and univalents, the range of different associations being 0-2VI, 0-5IV, 0-4III, 13-30II and $0-6 \mathrm{I}$ (Table 1). The average association was represented by $0.11 \mathrm{VI}+1.21 \mathrm{IV}+$ $0.18 \mathrm{III}+26.55 \mathrm{II}+0.86 \mathrm{I}$. Moreover, the multivalent pairing was maintained over the succeeding generations. In the amphiploid, each chromosome has a true homologue and it is reasonable to assume that bivalents in this hexaploid result from pairing of completely homologous chromosomes. The question arises as to why preferential pairing is much restricted in the amphiploid and how far bivalent pairing is influenced by the genetic control of chromosome pairing in A. hypogaea. If bivalent pairing alone was ensured in the allohexaploid by way of restriction of pairing to homologues, then the control system involved would be fit enough to distinguish between the chromosomes of the two $A$. hypogaea genomes and $A$. villosa although they possess pairing affinity. For such a system to be effective, a degree of differentiation would have to exist between the genomes of $A$. hypogaea and $A$. villosa.

In striking contrast, the allohexaploid of two wild species (A. villosa $\times A$. marginata) suffered from a reduction in the number of bivalents formed and an enhancement in the frequency of quadrivalents. Seventeen per cent of the cells exhibited 1-2 hexavalents, in fifty per cent, 5-10 quadrivalents occurred along with 10-20 bivalents and in 33 per cent, quadrivalent (4-9), trivalent (1-2) and bivalent $(10-20)$ associations could be found besides $1-2$ univalents. The mean association was $0.22 \mathrm{VI}+6.83 \mathrm{IV}+0.41 \mathrm{III}+14.81 \mathrm{II}+0.41 \mathrm{I}$. Only in 6.5 per cent of PMCs, 10 heterogenetic quadrivalents and 10 homogenetic bivalents prevailed.

It is assumed that duplication of the sets in A. hypogaea leads to preferential pairing notwithstanding the structural similarities between the two and the diploidised meiotic behaviour of the species is also reinforced by a genetic-factor located in a chromosome of the $\mathrm{B}$ genome. In the wild tetraploid ( $\left.\mathrm{A}^{\prime} \mathrm{A}^{\prime} \mathrm{BB}\right)$, however, quadrivalents prevailed and this points to the inactivation of the factor referred to by another residing in a chromosome belonging to the $\mathrm{A}^{\prime}$ genome. In the allohexaploid of the constitution $\mathrm{AAA}^{\prime} \mathrm{A}^{\prime} \mathrm{BB}$, the effect of regulation of preferential pairing of chromosomes is probably nullified by the $A^{\prime}$ genome and in the other amphitriploid with an autoalloploid genomic structure $\left(\mathrm{A}^{\prime} \mathrm{A}^{\prime} \mathrm{A}^{\prime} \mathrm{A}^{\prime} \mathrm{BB}\right)$, there was also a failure towards promotion of a bivalent regime. The cryptic structural differentiation between chromosomes of the genomes which showed gross homology in the triploid hybrids is not fully substantiated by the meiotic behaviour of the allohexaploids.

The origin of an octaploid plant (Fig. 20) in the natural population of $A$. hypogaea marked the single step event of natural hybridisation with a wild tetraploid species. The order of chromosome pairing in this high polyploid is not in consonance with its genomic formula of $\mathrm{AAA}^{\prime} \mathrm{A}^{\prime} \mathrm{BBBB}$. The maximum number of fourteen quadrivalents can be resolved as 10 autosyndetic ones provided by the B genomes and four others as arising from heterogenetic pairing between chromo- 

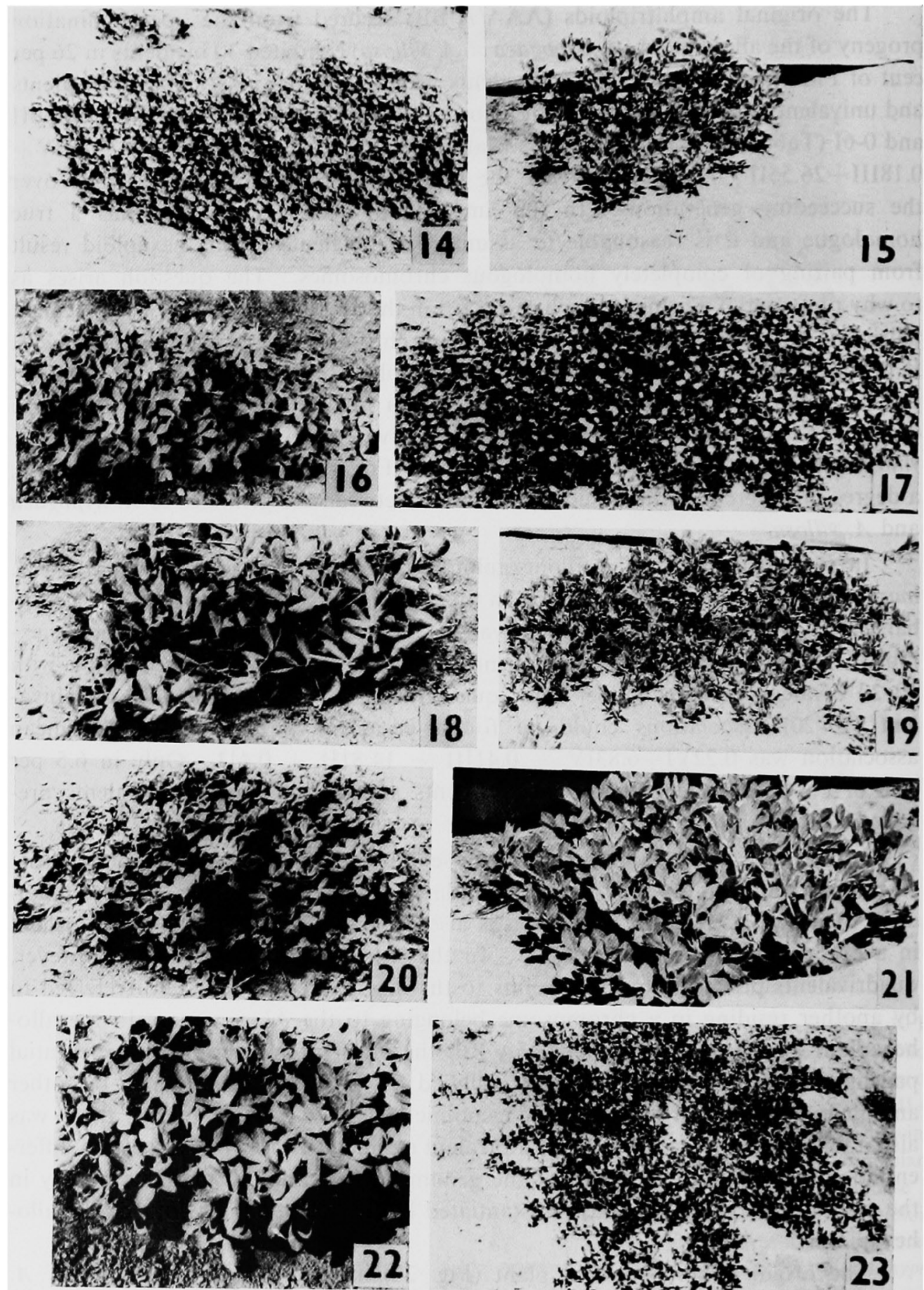

Figs. 14-23, 14, 15 and 16, triploid, tetraploid and pentaploid hybrids respectively of the parentage, $A$. hypogaea $\times$ Allohexaploid. 17 and 18 , tetraploid $F_{1}$ s resulting from cross pollinations of the natural allotetraploid (A. hypogaea) with the experimental autotetraploids of $A$. villosa and A. villosulicarpa. 19, hexaploid plant detected in the second generation progeny of the allotriploid ( $A$. hypogaea $\times$ diploid $A$. villosa). 20, octaploid seedling which occurred in the natural population of $A$. hypogaea. 21, tetraploid arising from reversion of the allohexaploid. 22, tetraploid in the back-cross progeny of $A$. hypogaea $\times$ Allotriploid. 23, triploid offspring of the tetraploid hybrid ( $A$. hypogaea $\times$ Autotetraploid $A$. villosa). Figs. 14, 15, 19, 21 and $23,1 / 8$ in reproduction and the rest, $1 / 6$. 
somes of the $\mathrm{A}$ and $\mathrm{A}^{\prime}$ genomes. Multivalents higher than quadrivalents occurred in 30 per cent of the cells. The pairing solely confined to the formation of quadrivalents and bivalents is not achieved even in the presence of the numerically strong $B$ genome assumed to carry the factor charged with the regulation of homogenetic pairing. The hybrid (AA'BB) between the annual ( $A$. hypogaea) and perennial tetraploid ( $A$. marginata) is equivalent to the polyhaploid of the octaploid plant. Eighteen per cent of the cells in the tetraploid hybrid had 20 bivalents, and in 9 per cent, the association was $18 \mathrm{II}+4 \mathrm{I}$. Seventy three per cent of the PMCs revealed quadrivalents (1-2), trivalents $(0-2)$, bivalents $(12-18)$ and univalents $(0-4)$. The maximum association of 2 quadrivalents and 2 trivalents existed in conjunction with 12 bivalents and 2 univalents. In tetraploid hybrids between species with the annual (A. monticola) and perennial habit (A.glabrata), 29 per cent of the PMCs revealed 1-3 IV, 0-2 III, 12-18 II and 0-4 I. Twenty bivalents figured in 29 per cent of the cells and the frequency of bivalents (17-18) plus univalents (4-6) was 32 per cent. The average association was $0.51 \mathrm{IV}+0.31 \mathrm{III}+17.36 \mathrm{II}+2.26 \mathrm{I}$.

The degree of differentiation among the component genomes is also evidenced from the meiosis of two allotetraploids, one derived from crosses between $A$. $h y$ pogaea and autotetraploid $A$. villosa and the other realised in the progeny of the cross, autotetraploid $A$. villosa $\times A$. hagenbeckii. The former with 1:2:1 proportion of the A, $\mathrm{A}^{\prime}$ and $\mathrm{B}$ genomes entailed the average association of 1.75IV + $2.60 \mathrm{III}+7.25 \mathrm{II}+10.60 \mathrm{I}$. The proportion of the $\mathrm{A}^{\prime}$ and $\mathrm{B}$ genomes in the other hybrid was $3: 1$ which gave an average pairing of $1.00 \mathrm{IV}+8.0 \mathrm{III}+1.50 \mathrm{II}+$ $9.0 \mathrm{I}$. The maximum frequency of heterogenetic multivalents was represented by 3 quadrivalents and 10 trivalents. Twenty per cent of the PMC's disclosed $10 \mathrm{III}+$ $10 \mathrm{I}$ and in twenty others, the pairing was represented by $3 \mathrm{IV}+7 \mathrm{III}+7 \mathrm{I}$. The order of chromosome pairing in the two hybrids brings out the cytological differentiation of the $\mathrm{A}$ and $\mathrm{A}^{\prime}$ genomes of the annul and wild tetraploids respectively.

The pentaploid is the product of hybridisation between $A$. hypogaea (TMV. 10 ) and the allohexaploid ( $A$. hypogaea $\times A$. villosa) and the dosage of the $\mathrm{A}, \mathrm{A}^{\prime}$ and $B$ genomes in the hybrid is $2: 1: 2$. Pairing was not confined to the combination of bivalents and univalents alone, and associations of 3 or more chromosomes existed leading to the range of $0-2 \mathrm{~V}, 0-2 \mathrm{IV}, 0-5 \mathrm{III}, 14-19 \mathrm{II}$ and $6-10 \mathrm{I}$. If preferential pairing was operative, the pentaploid need not have manifested associations higher than bivalents.

Diploid hybrids ( $A$. duranensis $\times A$. villosa) showed no evidence of gross structural differences between the chromosome complements of the parental species. But, the preferential autosyndetic pairing exhibited by the triploid possessing two genomes of $A$. villosa and one of $A$. duranensis is indicative of cryptic structural differentiation (Table 2). The allotetraploids of $2 \mathrm{n} \times 2 \mathrm{n}$ and $3 \mathrm{n} \times 2 \mathrm{n}$ origins also disclosed the differential affinity of members belonging to the two genomes. Small structural differentiation was expressed by way of precocious separation of bivalents at diakinesis in the meiosis of the diploid hybrid. The finer structural differentiation between the chromosome sets of the diploids is obvious, although their genomes presented gross homology with one of the two complements of the tetraploid species. 


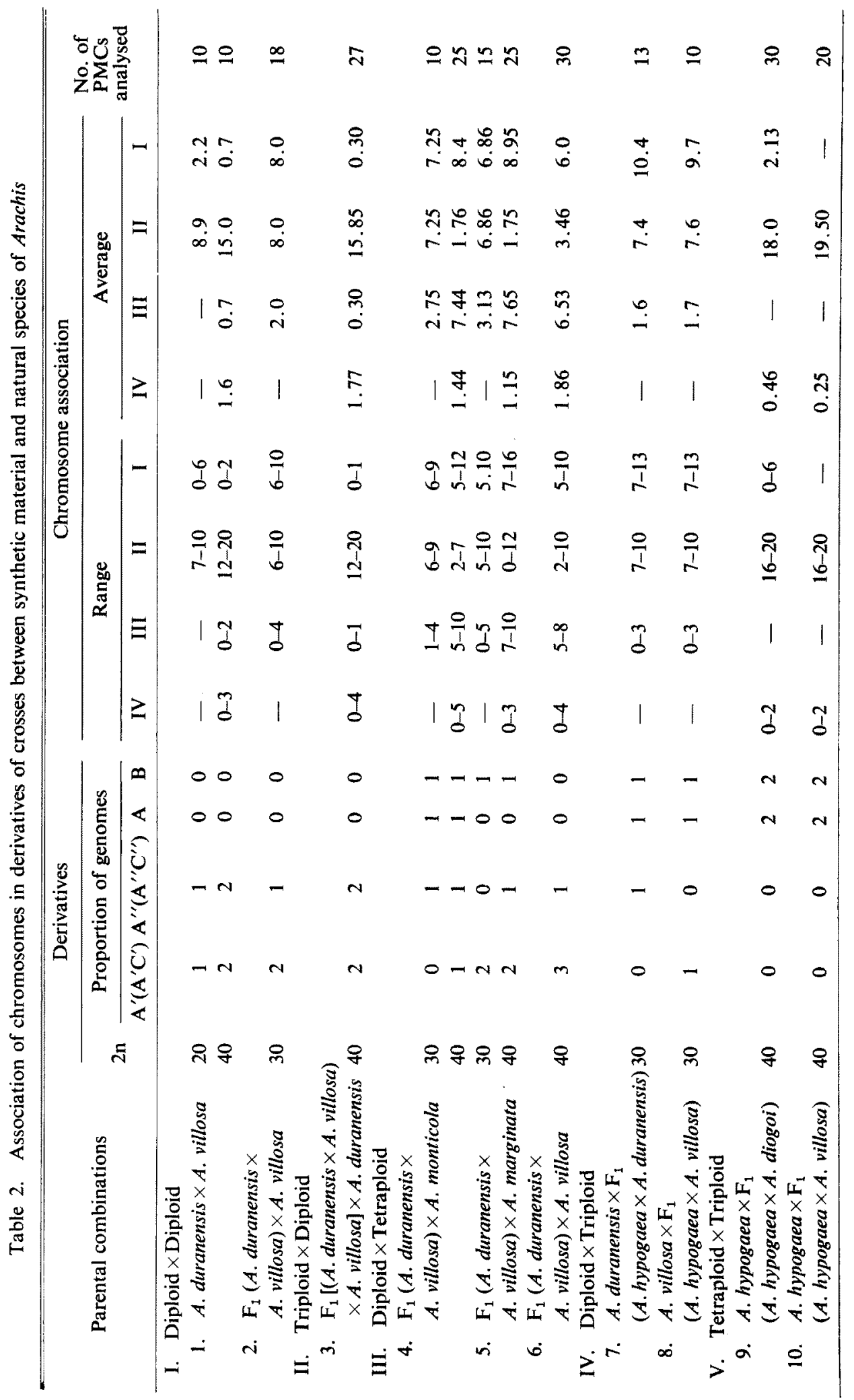


Cross-pollinations of the diploid hybrid with the annual (A. monticola) and perennial ( $A$. marginata) tetraploids enabled the recovery of tetraploid hybrids in the progenies. These hybrids were morphologically distinct but their meiotic chromosome pairing presented less recognisable deviations. In the organisation of the diploid egg cells, the two genomes of the diploid species are probably accomodated and the cytological mechanism by which it is accomplished is yet to be determined. It is also possible that one of the genomes may segregate preferentially for being included in the egg cell for eventual duplication. Thus, the deviation in the genomic content of the egg cells and the variation in the genotype of the pollen of the two kinds of natural tetraploids contribute to the cytomorphological expression of the tetraploid hybrids. Chromosome pairing in the tetraploid offspring of the parentage, diploid $F_{1} \times$ tetraploid $A$. villosa is not typical of an autotetraploid. It is quite probable, therefore, that the restituted egg cell of the diploid hybrid comprised the genomes of both the diploid parental species and in the genotype of the synthetic material, one genome of $A$. duranensis and three of $A$. villosa are present. The pattern of chromosome pairing between the two triploids viz., diploid hybrid $\times$ $A$. marginata and $A$. villosa $\times A$. marginata was not dissimilar. However, deviations in pairing behaviour though small in magnitude could be found between allotriploids of the parentage, diploid $\mathrm{F}_{1} \times A$. monticola and $A$. monticola $\times A$. villosa. Such a discrepancy can accrue from the participation of egg cells carrying the reconstituted genome of the diploid species in the origin of the former allotriploid, in contrast to the latter arising from the fusion of gametes, incorporating the pure genomes of $A$. villosa and $A$. monticola.

\section{Progeny behaviour of experimental polyploids}

The triploid of $A$. hypogaea (Spanish-POL. 2) with $A$. villosa, tetraploid hybrids of two Spanish groundnuts (TMV. 9 and TMV. 7) with the autotetraploids of $A$. villosa and $A$. villosulicarpa respectively (Figs. 17 and 18) and the allohexaploids (isolated in the open pollination progenies of triploid $F_{1}$ involving the Spanish cultivar TMV. 7 and two diploid species viz., A. duranensis and $A$. villosa) entailed varying levels of sterility, genic and chromosomal in origin. The second generation progeny of the allotriploid (AA'B) comprised $4 n$ and $6 n$ plants (Fig. 19) in the proportion of 1:5, whereas the allotetraploid ( $\mathrm{AA}^{\prime} \mathrm{A}^{\prime} \mathrm{B}$ ) yielded triploid (Fig. 23) and hexaploid offsprings, the latter comprising 99 per cent of the population. The percentage of occurrence of triploid, tetraploid (Fig. 21) and hexaploid seedlings derived from the allohexaploid (AAA' $\mathrm{A}^{\prime} \mathrm{BB}$ ) was approximately 1,29 and 70 . Chromosome pairing in the euploid descendants of the three experimental allopolyploids presented in Table 3 signifies the attainment of genomic balance promoted by selective fertilisation of gametes.

\section{Allotriploid}

The tetraploids resulting from the fusion of 20-chromosome gametes formed in the triploid resembled the cultivar in gross morphology but suffered from varying degrees of introgression of vegetative and pod characteristics of the diploid species. The morphological variation among the hexaploids can also be traced to the hetero- 


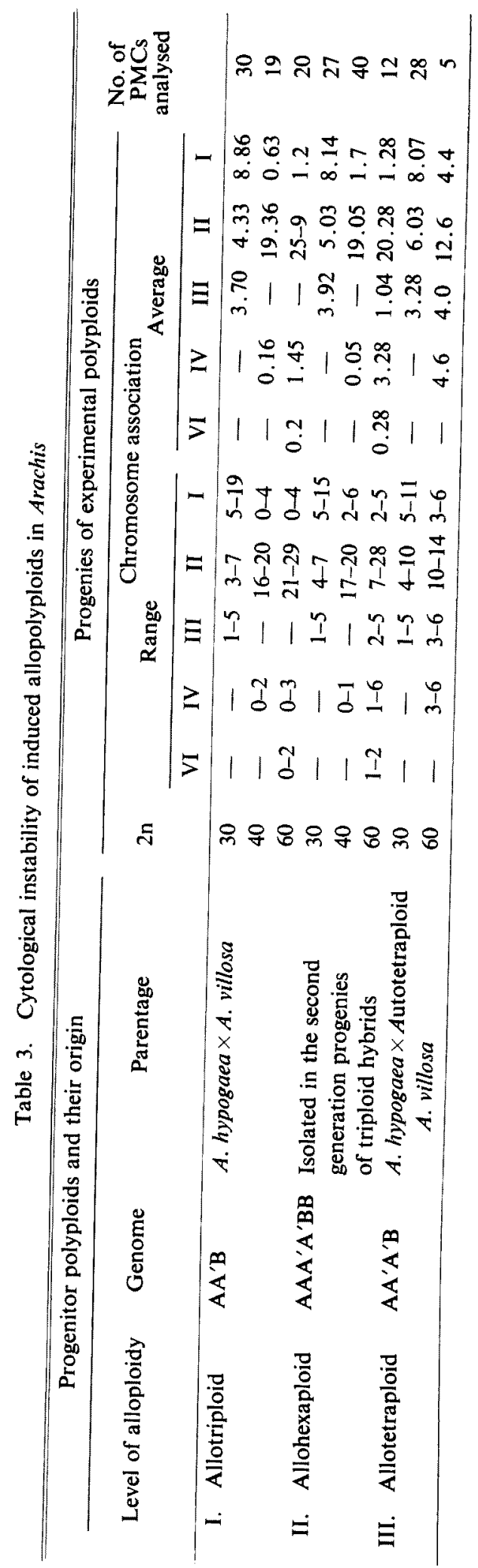


genetic pairing in the meiosis of the allotriploid and the consequence of such associations could be found in change of growth form, alteration and inhibition of flowering habit, and modifications in respect of features of the pod and kernel and level of productivity. A solitary triploid plant was also recorded previously in the second generation progeny of the allotriploid of $A$. hypogaea (Spanish-TMV. 7) with $A$. villosa. Tetraploid hybrids were secured in backcrosses of the Spanish cultivar (TMV. 7) and the Virginia-spreading groundnut (M. 13) with the two triploid hybridz viz., A. hypogaea $\times A$. diogoi and $A$. hypogaea $\times A$. villosa (Fig. 22). Diploid $\times$ triploid matings gave only triploid progenies (Raman 1976). The variation in the frequency of occurrence of balanced gametes with 10,20 and 30 chromosomes attained through directed genomic segregation in the meiosis of the allotriploid and the euselectivity of such gametes in fertilization probably account for the different proportions of euploid individuals in the progenies of triploid hybrids.

The occurrence of a $38(2 \mathrm{n})$ chromosome plant in the open pollination progeny of the allotriploid of $A$. monticola with $A$. villosa is indicative of occasional but successful participation of aneuploid gamete(s) in the origin of viable embryos (Varisai Muhammad 1973). In the monosomic plant detected in the X-irradiated progeny of a derivative of the hybrid between $A$. hypogaea and $A$. monticola, quadrivalents ranged from $0-1$, bivalents from 13-19 and univalents from 1-9. The average association was $0.26 \mathrm{IV}+17.53 \mathrm{II}+2.86 \mathrm{I}$. Forty per cent of the cells exhibited 19 II +1 I and in thirteen others, the association was represented by 1 IV +17 II $+1 \mathrm{I}$. In forty seven per cent of the cells, 2-8 univalents prevailed in addition to the chromosome lacking its homologue. The phenotype of the monosomic was not suggestive of the inclusion in its karyotype of whole chromosomes of the wild species. The additional univalents could have emerged either from precocious separation of such bivalents possessing a recognisable level of small structural variations or resulted from the phenomenon of univalent shift. The cytological behaviour was as it could be expected, the univalents alone contributing to the aberrant meiosis. The non-recovery of either monosomics or nullisomicsin the progeny speaks well for the fact that the deficiency is not tolerated in the haplophase. Arachis hypogaea and A. monticola are considered to be allopolyploids (Stebbins 1957, Raman 1964 and 1973). Yet the differentiation of the duplicated chromosomes appears to have advanced to a high degree to confer functional specialisation. The present case is different from that of the secondarily balanced allotetraploid of Oryza sativa, where the chromosome deficient plant $(2 n=23)$ yielded 1 nullisome, 2 monosomes and 1 disome in its progeny (Sampath and Krishnaswamy 1948). Univalents (2-6) and bivalents (16-18) were noticed in sixty per cent of the PMCs of a Virginiabunch groundnut with $38(2 \mathrm{n})$ chromosomes. Only in forty per cent of the cells examined, 19 bivalents alone existed. The nullisomic deviated from its normal counterpart in having shorter main stem and primary branches to a pronounced degree and the reduction in size of leaf and length of calyx tube and intensity of hairness was not spectacular. The breakdown of the chromosome-deficient microspores appears to be brought about at different stages and the stainability of pollen indicated the level of fertility as 67 per cent. Sterility of a greater magnitude on the female side probably contributed to the very low setting of pods and the shrivelled 
kernels drawn therefrom either did not germinate or gave rise to inviable seedlings. The reproductive potential of plants with 38 and 39 somatic chromosomes directs attention to tetraploidy as the optimum level for stability and the highly balanced genetic mechanism between the genomes of $A$. hypogaea.

\section{Allotetraploid}

The genomic constitution of the two secondary triploids was found to be similar to that of the primary allotriploid as evidenced from the pattern of chromosome pairing. Between the former two, small but recognisable deviations in external morphology existed and these were more sterile than the primary hybrid of $A$. hypogaea (POL. 2) with $A$. villosa. The hexaploids also had the balanced genomic constitution of AAA' $A^{\prime}$ BB. The cytological behaviour of the euploid descendants is a reflection on the nature of genomic segregation in the meiosis of the tetraploid hybrid and the selective fertilization of gametes. Tetraploid hybrids alone resulted in the backcross progeny of $A$. hypogaea with the allotetraploid (Spanish cultivarTMV. $9 \times$ autotetraploid $A$. villosa). In contrast, the tetraploid $\mathrm{F}_{1}$ of the cross, cultivated species (A. hypogaea-Spanish, TMV. 7) $\times$ autotetraploid $A$. villosulicarpa produced only tetraploid $\mathrm{F}_{2}$ progeny wherein controlled recombination was conspicuous. The restricted intergenomal pairing and expelsion of all the alien chromosomes in both the male and female meiosis are redeeming features. In tetraploid hybrids entailing the genotypes of $A$. hypogaea (Gangapuri, OSN. 1 and TMV. 3) and the autotetraploid $A$. villosa and $A$. villosulicarpa, intergenomal pairing did not conform to the same model as also the pattern of organisation of gametes and their preferences in fertilisation.

\section{Allohexaploid}

The recovery of $3 n, 4 n$ and $6 n$ plants in the selfed progeny is a pointer to the reproductive instability of the allohexaploid. The triploid was similar in its cytomorphology to the allotriploid isolated in the progeny of the tetraploid hybrid ( $A$. hypogaea $\times A$. villosa). Three kinds of gametes differing in chromosome number and constitution resulting from genomic segregation in the meiosis of the hexaploid advanced the origin of the $3 n, 4 n$ and $6 n$ plants through selective fertilization. The limited variation acquired by the reverted tetraploids is a sequel to the restricted segmental homology of the pairing partners in the meiosis of the hexaploid. The differences in both the number and other characteristics of the pod of the tetraploids is a more prominent aspect of introgression. The hexaploid seedlings assumed new expressions for the habit of growth and consisted of two sorts viz., the flowering and non-flowering; seed-sterile and seed-fertile plants comprised the former. Vegetative luxuriance, longer growth period, inconstant levels of expression for productivity and diminution in size of pod and kernel were the properties displayed. Hexaploid plants combining accetable growth periods with reasonable size of kernel and level of pod yield should also enjoy the diploidised meiotic behaviour. Backcrosses of $A$. hypogaea (OSN. 1, TMV. 9 and TMV. 10) with the hexaploid resulted in 3n, $4 \mathrm{n}$ and $5 \mathrm{n}$ offsprings (Figs. 14-16). Despite the possesion of duplicated genomes, the allohexaploid displayed cytological instability, but this disability has not engendered its total breakdown. 


\section{Discussion}

The lack of major abnormalities at diakinesis and later stages of meiosis in hybrids between $A$. hypogaea and $A$. monticola revealed the genomic homology of the two species, though the lesser frequency of chiasmata signified structural differentiation. Differences between the two species appear to be largely genic superimposed by small structural differences of low order as evidenced from the pattern of segregation for fertility and interspecific differences in the $F_{2}$. Loosely paired bivalents are more prominent in hybrids between the cultivated and truly wild groundnuts and the skewness in distribution of $F_{2}$ progenies provided evidence for structural inequalities which are conducive to the non-recovery of certain recombinants due to altered linkage relationships and selective expelsion of gametes and zygotes; diplontic sterility factors also exercise their influence to impart higher levels of sterility in the $F_{2}$ plants compared to that noticed in the hybrids. The diploid hybrid also suffered from a small reduction in fertility, in contrast to the parents and a restriction in recombination for the morphological traits which distinguish the parents. The meiotic behaviour of the segregants was similar to that of the hybrid and the former being relatively fertile.

The affinity between the complements present in the hybrids is well exhibited as to allow of pairing between most of the chromosomes of the parental genomes. The preferential pairing noticed in the natural polyploid was lessened in the experimental ones and the differential affinity also could exercise a gentle assertion. Two factors can be responsible for this behaviour. First, there is segmental homology between the genomes, the pairing regions being limited mostly to the distal portions of the chromosomes and secondly there is a genetic mechanism which enhances the expression of affinity. The presence of loose pairing in bivalents and the end to end associations in multivalents lend support to the first. The occurrence of univalents and distinctive manifestation of associations at the successive stages in the first division suggested differential affinity and the attendance of factors which may be of genetic nature causing an inhibition of preferential pairing is obvious from the encouragement of multivalents at diakinesis. It is probable that the genotype of the diploid species of Arachis has a decisive influence in suppressing the diploidising effects produced by genes in the artificial allopolyploids.

It is evident that a diploid species similar to $A$. villosa has contributed the A genome to the present day cultivated species and the $B$ genome present in the tetraploid may be completely alien to the first or constituted of parts or segments which have been differentiated in the course of evolution. The high fertility coupled with the strong secondary association of chromosomes at metaphase in the diploids points to their allotetraploid origin. Three diploids, $\mathrm{AACC}, \mathrm{A}^{\prime} \mathrm{A}^{\prime} \mathrm{C}^{\prime} \mathrm{C}^{\prime}$ and $\mathrm{BBC}^{\prime \prime} \mathrm{C}^{\prime \prime}$ are assumed to be ancestral to the present day tetraploid species of Arachis, the complement of the first two alone possessing a pair of smallest chromosomes (Achromosomes of Husted). Hybridisation followed by chromosome doubling could have resulted in the establishment of the annual (AACC/BBC ${ }^{\prime \prime} \mathrm{C}^{\prime \prime}$ ) and perennial $\left(\mathrm{A}^{\prime} \mathrm{A}^{\prime} \mathrm{C}^{\prime} \mathrm{C} / \mathrm{BBC}^{\prime \prime} \mathrm{C}^{\prime \prime}\right)$ tetraploids $(2 \mathrm{n}=8 \mathrm{x}=40)$. Thus, the 10 chromosomes of the $A$ genome of the annual tetraploid will comprise the subgenomes of $A$ and $C$ with 
5 chromosomes each and the B genome in turn will be constituted of the subgenomes, B and C." The putative parents of the present day tetraploids have some segmental homology as indicated by the formation of trivalents at meiosis in the triploids and trivalents without corresponding number of univalents in autotetraploids of diploid species. In allotriploids, it is common to find 5 of the univalents showing delayed movement and division while the other move to the poles along with the chromosomes which had synapsed. This fact viewed in conjunction with the secondary pairing in diploids points to the basic chromosome number as $5(\mathrm{x})$.

The presence of a pair of smallest chromosomes is conspicuous in the karyotype of the diploid and tetraploid species of Arachis thus far investigated. Smartt et al. (1978) have reported the existence of $A$. batizocoi whose complement is devoid of the smallest pair of chromosomes. Based on morphological and phytogeographical evidence, the authors believed that the genome donors of the tetraploid are the A. batizocoi diploid species of $A$. cardenasii and A. batizocoi.

The breeding behaviour of induced polyploids of Arachis has brought to light the possible pathways for introducing genetic variation from the diploid to the tetraploid notwithstanding the impediments encountered in experimental introgression. Post-fertilization barriers of different intensities existed and restrictions on recombination and variation are imposed by haplontic and diplontic sterility factors. Intergenomal pairing was not fully suppressed and auxiliary mechanisms facilitated the directed distribution of genomes. Selective fertilization of gametes also prevailed thereby favouring the origin of polyploid progenies. Tetraploidy appeared to be the optimal limit for breeding new groundnuts as exemplified by the synthesis of tetraploid genotypes of $A$. hypogaea incorporating specified chromosomal segments of $A$. villosulicarpa through controlled recombination (Raman 1978).

\section{Summary}

Studies of chromosome pairing in interspecific hybrids and induced allopolyploids of Arachis elucidated the genomic structure of $A$. hypogaea and other tetraploid species. The diploid species $(2 n=20)$ probably originated from 10 chromosome ( $2 \mathrm{n})$ ancestors as yet unknown. In view of the paucity of evidence from studies on diploid hybrids and their amphidiploids, the nature of genomic differentiation of the individual diploid species is not well understood at present. The chromosomes of the haploid complement of the diploid species have homeologous counterparts in the A and B genomes of the tetraploid species and a set of 5 chromosomes in one of the genomes of the latter appears to exhibit a high level of differentiation. In the allohexaploids which comprise the genomes of the tetraploid and diploid species, pairing seems to be enhanced by genetic mechanisms even to give maximum expression to the residual affinity. It is probable that the genotype of the diploid species brings in the accentuation of pairing due to a breakdown of diploidising factor present in the polyploids of Arachis.

Triploid and tetraploid hybrids and the allohexaploid manifested multivalent chromosome pairing and aberrant meiotic behaviour. The realisation of balanced euploid derivatives in different proportions signified the organisation of gametes 
$\mathrm{h}$ three different chromosome numbers as a result of directed genomic segregaa in the meiosis of the progenitor polyploids. Euselectivity of gametes in ferzation was also evidenced from the analysis of progenies resulting from backcses of $A$. hypogaea with the three synthetic polyploids. Auxiliary mechanisms sing from genomic combinations seemed to be effective in determining the breed; behaviour of the experimental polyploids. The deviations in the strength of ression of the genic mechanisms also appeared to depend upon the differences in : genomic composition of the interspecific hybrids and induced polyploids.

\section{Acknowledgement}

The information and data provided in the article are drawn from investigations the cytogenetics of cultivated groundnuts ( $A$. hypogaea) and related species of achis under the All India Co-ordinated Research Project on Oilseeds carried out ring 1971-79.

\section{References}

man, V. S. 1964. Progress of cytogenetic research in Madras State. In Advances in Agricultural Sciences and their Applications. Pub. Madras Agricultural Students' Union, Coimbatore, India pp. 122-143.

1973. Genome relationships in Arachis. Oleagineux 28: 137-140.

1976. Cytogenetics and Breeding in Arachis. In Aspects of Plant Science, Vol. II, pp. 1-84, Pub.: Today and Tomorrow's Printers and Publishers, New Delhi.

1978. An instance of success in experimental introgression of wild germ plasm into the cultivated groundnut (Arachis hypogaea). Paper contributed to the Summer Institute on "Breeding Methodology in Pulses and Oilseeds" organised by the Indian Council of Agricultural Research at the Punjab Agricultural University, Ludhiana during June 1978. mpath, S. and Krishnaswamy, V. 1948. A chromosome deficient paddy type. Curr. Sci. 17: 271 .

sartt, J., Gregory, W. C. and Pfluge Gregory, M. 1978. The genomes of Arachis hypogaea I. Cytogenetic studies of putative genome donors. Euphytica 27: 665-675.

zbbins, G. L. 1957. Genetics, evolution and plant breeding. Indian J. Genet., 17: 129-141. rrisai Muhammad, S. 1973. Cytogenetical investigations in the genus Arachis II. Triploid hybrids and their derivatives. Madras agric. J., 60: 1414-1427. 